\title{
THE IMPACTS OF STUTTERING SHIFTS TO THE TRANSLATION QUALITY OF THE ADVENTURE OF TINTIN COMICS
}

\author{
Rudy Prasojo \\ roedy.prasodjo@gmail.com \\ Universitas Sebelas Maret Surakarta
}

\begin{abstract}
This article aims to discuss how stuttering utterances are translated from English to Bahasa Indonesia. Data in the form of stuttering utterances spoken by Captain Haddock, a character in The Adventure of Tintin comic series, were collected to see how the translator decided to convert those utterances into Bahasa Indonesia. The method employed in this research is the qualitativedescriptive in which the data were taken from two sources(English and Bahasa Indonesia). The finding shows that there are three categories of stuttering initiator namely: intoxicated, emotions, and situations. Each category is classified based on its types, namely repetitions, hesitations, and fillers. In addition, the finding also shows that each type of stuttering was placed in a different concept on its target language named shifting. It can further be classified into preserved, altered, and omitted. Each of them affects the meaning and the message of stuttering utterances in the translation version. Finally, the statistic indicates that every type of shifting gives a significant impact to the translation quality.
\end{abstract}

Keywords: stuttering shift; Captain Haddock; translation quality

\section{INTRODUCTION}

Stuttering is a speech disorder characterised by repetitions, prolongations and blocks that disrupt the forward movement of speech (Belyk, Jo \& Brown 2014). Stuttering can be defined as the loss of control over the speech that someone wants to say because there is a disturbance between cognitive and the instrument of his speech which in turn produces in stammered speech.

On the other hand, Gruyter (2018) states that a stuttering can be described as a 'choral, composition based on the exchange of alphabets, where language has been stripped back to its basic building-blocks letters, symbols and sounds.It has often beenobserved that stuttering changes from time to time, from situation to situation (Alm 2014).

First research about stuttering was presented by Johnson (1959) through an experiment to analyze the characteristics of the stuttering formstowards some respondents who have different backgrounds. One of the results of his study is the invention of a type or category of stuttering that appears based on its forms, including prolonged sound, repetitions, interjections (pauses: between words or sentences, such as: uh, er, um), and revisions. Furthermore, stuttering form category is the subsequent studies, such as Miller et al. (2005) who defined stuttering as part of language disability (linguistic disfluency) due to difficulties in finding words, which not only occurs in stutterer (person who suffered stutter from childhood) but also in normal people (could be influenced by various factors. Guitar (2014) suggests that, one of factors causing speech stuttering is a psychological factor which can be categorized in to two types, namely cognitive and affective factors. The cognitive factor is caused by brain disfunction that occurs due to both congenital defects and temporary brain function disorder. Hollien \& Martin (2001) describe that intoxication 
caused by alcoholic liquor or maybe drugs causes the speaker to say more often stutter. Moreover, affective factors are divided into two subcategories. The first subcategory is emotions (emotional level), which is a visualization of theoutburst of the speaker's feeling (happy, angry, sad, etc.) which is generally caused by external factors or other person. The second is situations which can be described as the environment or condition which causesan individual to experience stuttering.

In relation to comics, McCloud (1993) explains that comic is a literature that consist of pictures, and writings or narratives which tell the stories simultaneously and presented side by side (juxtaposed). Therefore, in comics and illustrated books, there is always a connection between narrative and images. Thus, in translating a comic, the translator should pay attention to the style of the author and the text, because it is not only a matter of delivering the messages but also building the characteristics of comic and all elements in it.

At some point, the stuttering words which are spoken by Captain Haddock experience changes or shifts in the Indonesian translation, in which the shift is in the form of place shifting. For instance, there is a shift of the stuttering from the subject in the source language (SL) that positioned changes to a verb or even an object in the target language (TL). This certainly effects the sentence meaning and the message occured in the translation version. According to Catford (1965), a translator must know the basic techniques in solving translation through shifting meaning and form in general. Nida \& Taber (1982) explains that translation is the process of producing the equivalent meaning closest to the message, where the sentence's composition and meaning must be in equal (both SL and TL).This is in line with Nababan \& Nuraeni
(2012) which also suggest that translation is a process of delivering messages from the SL to the TL as naturally as possible.

In addition, Nababan \& Nuraeni (2012) suggest that the quality of a translation can be categorized into three types, namely: accuracy, acceptability, and readibility. The translation can be said to be ideal if the message accurately delivered from the SL to TL is, the sentence is acceptable or in accordance with TL rules and the translation are easily understood by the target reader. Nababan et al. (2012) proposes that to measure the quality of translation requires three aspects of assessment which include the accuracy of message transfer, the accuracy of the disclosure of messages, and the nature of the translation language. However, there are many cases that translators face the choice of giving priority to choose one aspect at the expense of the other two aspects. Therefore, in reality, it is very difficult to find a flawless translation that consists of all aspects.

Here are previous studies that discuss about stuttering or linguistic disfluency in the field of translation. First, Tissi (2000) suggests that stuttering in the TL always has a bond with the SL. Therefore, as much as possible stuttering must be transferred to the TL as a form of identity even though it has a different concept. This finding supports the hypothesis that this types of stuttering are always found in varieties of hesitations and repetitions in every simultaneous interpretings. Second, Bakti (2008) examined the problems that are often faced by an interpreter in finding lexical and grammatical equivalents in Stuttering. Third is a study by Plevoets (2016) which discussed how the speech of stuttering in the form of interjection $(u h, m)$ in the SL is diverted by the number of more repetitions in the TL. And the last is the research of Hough et al. (2016) who examined the form of disfluency in multi languages as a form 
of speaker identity that must be well maintained by the interpreter.

From those previous studies, stuttering analysis in all of them only occurrs in the form of simultaneous interpretation. Although it has touched the translation aspect, but all of them do not discuss comprehensively about the impact of the stuttering shift in the translated text version. Generally, the entire study only presents types of stuttering which were calculated based on the frequency of occurrence of each category found. This is an interesting issue to be used as a research topic because stuttering is not only a speech phenomenon that usually used in a such a scheme, but also found in many literarture forms that have the translated version. Hence, beside of having to pay attention to the meaningfulness between the SL and TL, the stylistic effect of the stuttering's translation and the target reader ability to acknowledge the text also need to be considered in order to produce an ideal translation quality. Therefore, this research was conducted with the aim to see the impact of stuttering shifts presented by Captain Haddock in The Adventure of Tintin comic series on the quality of the Indonesian translation.

\section{METHODOLOGY}

This is a research in translation with a descriptive-qualitative approach method with single case for academic purposes as formulated by Sutopo (2002). It is a qualitative category, in which data are collected in the form of words, sentences, or pictures and not in the form of numbers. This research includes a single case study because the target or subject has the same characteristics.

The main objective of this research isnot to answer the hypothesis but rather to reveal the impact of the stuttering shift spoken by Captain Haddock in The Adventures of Tintin comic series to the translation version.
The location in this study is the material or reference media used in conducting research. According to Spradley (1980) the main elements of the research location can be divided into three types. First, the setting or place in this study refers to The Adventure of Tintin comic series in English and Indonesian version. Secondly, the participant, in this study was the Captain Haddock as a figure of stutterer. Third, the event taken, in this study is several editions from The Adventure of Tintin comic series and its translations, namelyThe Crab With The Golden Claw, Tintin in Congo, Red Sea Shark, and Red Rackham Treasure.

The data are in the form of speech acts which contain the terms or forms of stuttering. The context of this study is the visualization of images presented along with the text of stuttering. It is used to classify the stuttering category based on the initiator factors.

The technique of data collectionconsists of three categories, they are: content analysis, questionnaire, and focus group discussion. The validity of the data was carried out by intensive examination of the forms of stuttering shift mapping occurred in the Indonesian version. The examination was carried out by utilizing the credibility of three raters who mastered linguistics and translations.

At the analysis phase, the first step is to analyze the SL text by reading it in an integrated manner to understand the contents of the text (Nababan 2008, 26). In domain analysis, data was collected based on the initiator category of stuttering occurrence spoken by Captain Haddock. In taxonomic analysis, the findings are sequentially aligned between the SL and TL to determine and explain the form of shifts that occur. Next, a compound analysis, according to Santosa (2003), is the impact of the relationship between the two categories (domain and taxonomy) which is reduced to the third element 
(translation quality) which could be used to answer the problem formulation of the study.

\section{FindingS}

This section presents the result of this research in the form of finding stuttering initiator categories as well as a form of stuttering shifting spoken by Captain Haddock in The Adventure of Tintin comic series found in SL.

\section{Domain Based on Stuttering's Initiator}

The first is about domain determination that is based on the initiator of stuttering spoken by Captain Haddock presented through image visualization and the form of stutterings (e.g. repetitions, hesitations, filler, and revisions). For detailed information, see Table 1.

Table 1. Stuttering's Initiators

\begin{tabular}{lcc}
\hline Domain & Number & Percentage \\
\hline Drunks & 125 & 75,75 \\
Situations & 30 & 18,18 \\
Emotions & 10 & 6,06 \\
\hline
\end{tabular}

\section{Drunks (intoxicated)}

The first domain is intoxicated or easily recognised as drunk which causes the character to experience transient brain damage or in other words temporary cognitive impairment which initiates stuttering presented by Captain Haddock. It can be easily seen from image visualization, where Captain Haddock is under influence of liquor and speaks stutter. From the table above it can be seen that this category ranks at the top based on the number of data findingabout $75.75 \%$ of total data. See the illustrated text examples in Figure 1.

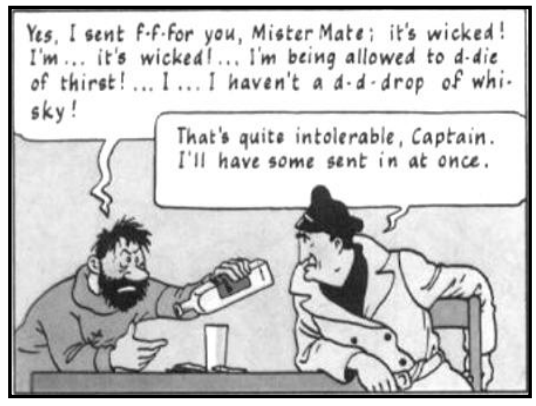

Figure 1: Stuttering category of drunks

From the example of Figure 1, it appears that Captain Haddock is holding a bottle of liquor. It is clear that the context of the conversation in Figure 1 shows Captain Haddock is drinking and getting drunk. Stuttering forms that appear in Figure 1 include; repetitions, that is the repetition of the prepositional phrase where the first letter in the word 'for' is repeated three times to 'f-f-for' and a word repetitions at 'I ... I'. Then there are also other stuttering forms, namely the revisions found in the phrase 'I ... it's wicked!'.

\section{Situations}

As mentioned before where situations isan environmental conditions that affect the character's speech tostutter, it usually occurs due to natural factors that make the character become hesitant or confused which has an impact on his speech that experiences stuttering. This category appears at the second after intoxicated, where around $18.18 \%$ is found in the dialog spoken by Captain Haddock that occurs in several parts of the comic series. For more details, see Figure 2.

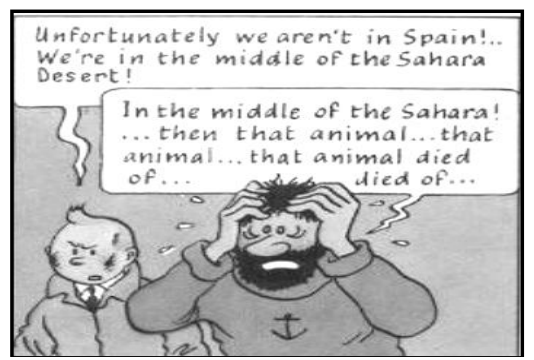

Figure 2: Stuttering category of situations

From Figure 2, it can be seen that stuttering that occurs is a form of repetitions of phrases found in the 
words "that animal ... that animal ..." and "died of...died of...". Stuttering initiator in the figure 2 are explained by the background of location where the dialog took place that they were stranded in the middle of desert.

\section{Emotions}

The category of emotions in this research is an overflowing feeling that caused Captain Haddock's speech becomes stutter. Emotions category are identical to some influences caused by other characters (speech partners). This category took place in the third position with the percentage of data appearing around $6.06 \%$ (see Table 1). Explanation of this category can be seen in Figure 3.

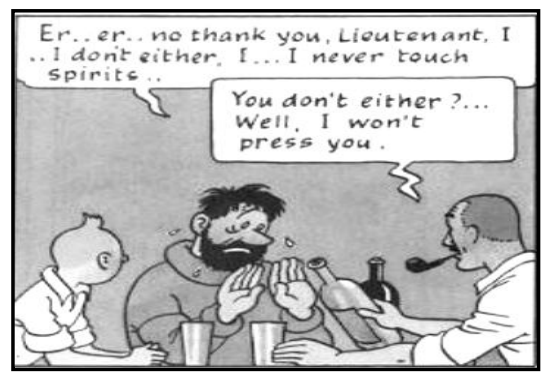

Figure 3: Stuttering category of emotions

From the example of Figure 3, it can be seen how the character of Captain Haddock is portrayed as a drunkard who tries to refuse an offer of a drink, thus experiencing an inner conflict or psychological disorder that causes his speech to experience stuttering. In the example of figure 3 can be seen two categories of Stuttering forms which are shown through the expression 'Er ... er' which is a form of hesitations, and there is a form of word repetition in 'I ... I.'.

\section{Stuttering Style in Translated Version}

Stepping on the second section, the stuttering style discussion is about how the stuttering form is shift-translated into Indonesian version. The shift in this study focused on the form of shifting places from SL into its TL. This is because the form of stuttering in the dialog is a form of sylistic effect (author writing style) in describing the characteristics of Captain Haddock. So how the characteristics of the stuttering writing style in the dialog spoken are transferred into the TL clearly affects the aspects of translation quality. The appearance of these shifts can be seen in the presentation of Table 2.

Table 2: Stuttering's Shift Mapping

\begin{tabular}{lrc}
\hline Shift Map & Number & Percentage \\
\hline Altered & 103 & $62,42 \%$ \\
Preserved & 33 & $20,00 \%$ \\
Omitted & 29 & $17,58 \%$ \\
\hline
\end{tabular}

As presented on Table 2, it can be seen if the stuttering that experienced a shift was 103 out of a total of 165 findings or around $62.42 \%$. The altered means the stuttering forms were replaced in other words format in the target text. Then, for Stuttering that did not experience a shift were found about 33 data or about $20.20 \%$. And the stuttering that omitted in the target text were found arround 29 data or about $17.58 \%$.

The details about each categories can bee seen in the explanation below.

\section{Altered}

In this category, the stuttering style spoken by Captain Haddock experiences a place shifting in the grammatical structure or it could also be stuttering shift on the basic sentence pattern. For more details, see Figure 4.



Figure4: Altered and preserved stuttering

The last sentence in the Figure 4, there is a form of displacement in the sentence 'in my hold...m-m-mine?' translates to Di-di-di gudang saya?. Stuttering in the form of repetitions occurred in the pronouns on SL shift its 
place to lie in the preposition on TL. The sentence pattern of the two languages is different and so it is called the Stylistic effect. This shift is slightly reduced the accuracy of the meaning and messages contained in the source language.

\section{Preserved}

In this second category, stutterings spoken by Captain Haddock do not experience changes or are still translated accordingly to the style or characteristics of lexical or grammatical structures that are appropriate to the context of SL. In Figure 4 it can be seenstutteringin the word 'O-o-opium?' which is a form of repetition maintained by the translator into Ga-ga-ganja? in the translated text which is a form of repetition also. As shown by Figure 4, it is clearly shown that the translator wants to keep maintaining the characteristics of Captain Haddock who is experiencing a psychological disorder called stuttering.

\section{Omitted}

The last category is the omission of the stuttering style in the TL. Furthermore, the omission of the stuttering style can occur due to the translator's decision that does not divert it, oreven the whole sentences have been deleted. Of course, it has an impact on the value of the translation quality in the TL. The example can be seen in Figure 5.

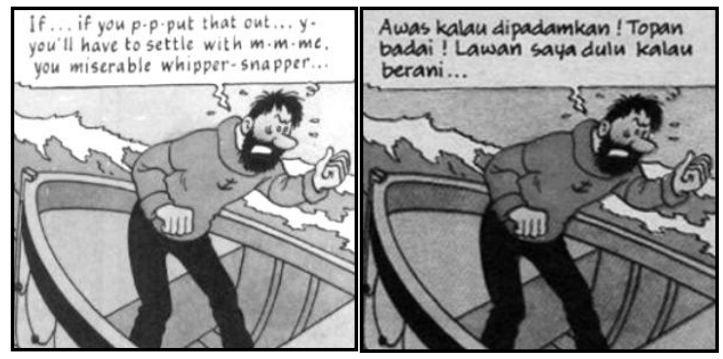

Figure 5: Omitted stuttering

In Figure 5, stuttering style are not translated or omitted in TL. Thus, the text spoken by Captain Haddock does not indicate that he is in a psychological disorder or experiencing stuttering.

\section{Stuttering Translation Quality}

The third section is about translation quality. Translation quality can be divided into three categories, namely (1) accuracy, (2) acceptability, and (3) readability. These three quality aspects can be seen in the presentation of Table 3 .

\section{Accuracy}

Accuracy has three value levels or scales as a measure of the translation quality, namely: accurate, less accurate, and inaccurate. The rating scale for the accuracy aspect in the presentation of table 3 shows that $65.93 \%$ of the translations have accurate values. While about one third or 29.63\% indicates that the translation results are less accurate. Finally, only 6 data from a total of 165 data showed that the translation is inaccurate. Each of its categories will be discussed in the explanation below.

Table 3: The Quality of Translation

\begin{tabular}{llrcc}
\hline & Quality & Num. & Pct. & V \\
\hline A & Accurate & 103 & 65.93 & 2.61 \\
& Less accurate & 33 & 29.63 & \\
& Inaccurate & 29 & 4.44 & \\
Ac & Acceptable & 136 & 67.41 & 2.65 \\
& Less & 24 & 31.11 & \\
& acceptable & & & \\
& Unacceptable & 5 & 1.48 & \\
R & High & 150 & 96.30 & 2.94 \\
& Medium & 10 & 2.22 & \\
& Low & 5 & 1.48 & \\
\hline
\end{tabular}

Accurate

First, the idea of accurate is the utterance of the sentence translated entirely whether the stuttering marker, phrase or clause in the sentence is transferred accurately into the target language without any reduction or addition in it. See Figure 6.

All the linguistic elements in Figure 6 spoken by Captain Haddock, whether the stuttering style or the sentence structure are correctly translated. There is no reduction or addition of any 
linguistic elements in the sentence, so that the translation is accurate as same as what occured in SL.

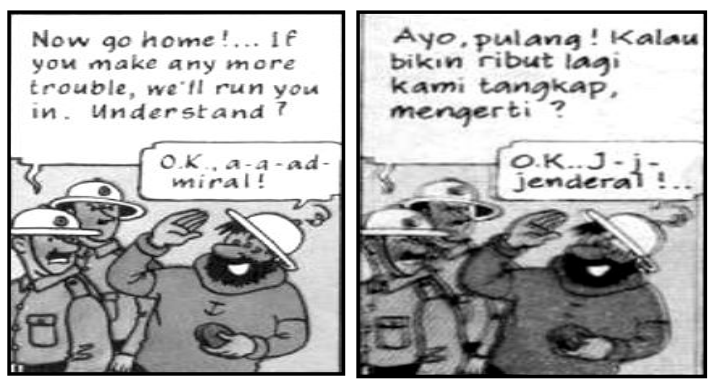

Figure 6: Accurate translation

Less accurate

A translation is categorized as less accurate if some of the meaning of the word or technical term in the text changes or is not accurately translated into the TL, thus reducing the integrity of the meaning and message contained in the whole sentence. See the example at Figure 7.

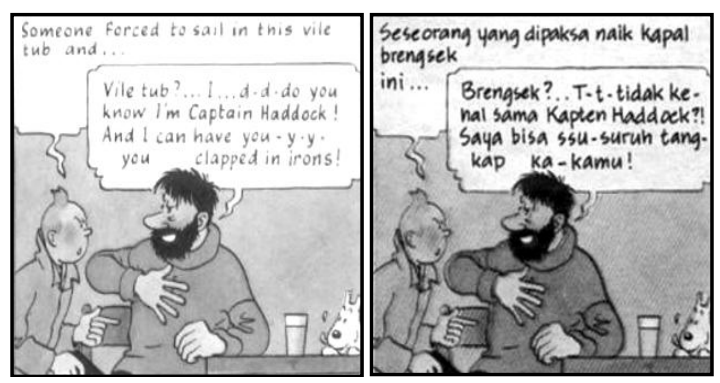

Figure 7: Less accurate translation

In the last sentence there is an addition of stuttering style, where in the SLit is only found in the word 'You$y$-y-you' experiencing a change in the TL to be located in the words 'ssu-suruh' and ' $k a$-kamu'. This decision results in a distortion of meaning and, of course, reduces the accuracy of whole meaning and messages occured in the SL text.

\section{Inaccurate}

The translation is categorized as inaccurate because the transfer of meaning and message of TL is not in match with the meaning from the SL, whether it is a reduction, misinterpretation or deletion of the linguistic elements or even the whole sentences. See the example in Figure 8.

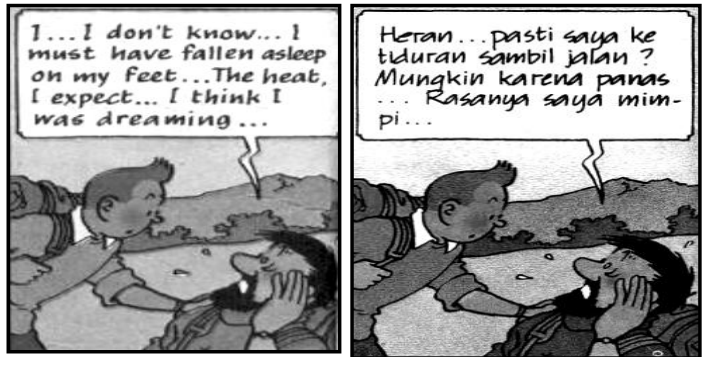

Figure 8: Inaccurate translation

The first sentence that contains stutteringstyleat Figure 8 , in the words 'I ...I don't know' turned into the word heran in TL has a very different concept of meaning. The stuttering style in the dialogue spoken by Captain Haddock is omitted at the SL text, and so the message that he is getting a psychological disorder in the dialogue is dismissed.

\section{Acceptability}

Acceptability is the second aspect in assessing the quality of translation in this study. The assessment criteria for this aspect are the same as the first aspect, where acceptability also has three value level scales as a measurement of the quality of the translation, namely acceptable, lessacceptable, and inacceptable. From Table 3, it can be seen that the acceptable findings have a value of 136 data from a total of 165 data. This means that speeches that contain stuttering styles spoken by Captain Haddock as much as $67.41 \%$ can be accepted or in match with the grammatical complexity of writing in Indonesian language. Only few of the findings are categorized as less acceptable and unacceptable. Each of those categories will be discussed in the explanation below.

Acceptable

An acceptable translation can be interpreted that the TL stuttering utterance has a grammar that is in match with Indonesian language rules, norms and culture. Or, it can also be the stuttering style on SL and TL text has the same concept or arrangement, without regarding the accuracy of the 
meaning. The example can be seen in Figure 9.

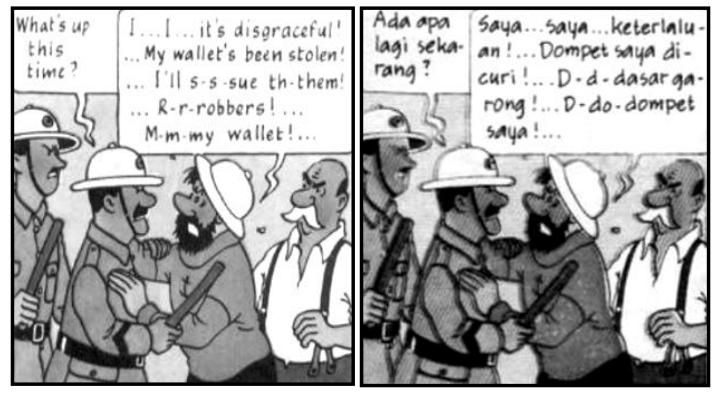

Figure 9: Acceptable translation

Focusing on the sentence '... Mm-my wallet!..' translated to D-do-dompet saya!.., there is a shift in the stuttering style, from preposition to head, but the phrase structure is correct according to Indonesian grammar, and so the stuttering style is still maintained. Thus, it becomes an acceptable translation.

Less acceptable

Less acceptable translation means that the translation results are less natural or not in accordance with good and correct Indonesian writing rules. In this study, The shift of the stuttering style that does not match the sentence pattern in the TL or some cases there is a pure borrowing foreign terms causes the utterance to be less acceptable. The clear explanation can be seen in Figure 10.

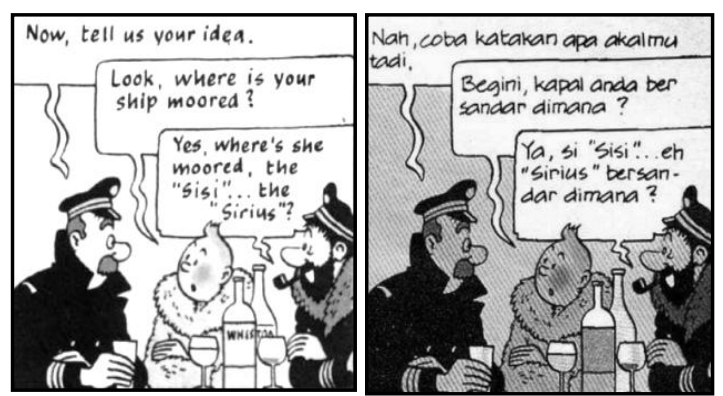

Figure 10: Less Acceptable translation

In Figure 10, the utterance 'Sisi'... 'Sirius'.." is a foreign term borrowed directly without any changing in its lexical structure. The word 'Sirius' is the name of a ship in the comic story where the translator makes a pure borrowing of the term because there is no word equivalent to certain terms that refer to the naming of humans or things. The presence of foreign linguistic elements in the sentence structure in the Indonesian translation text above results in translation that is less acceptable.

\section{Unacceptable}

Unacceptable translation means that the structure in the translated text is different from the TL structure, or the text is not translated and even deleted. The details can be seen in Figure 11.

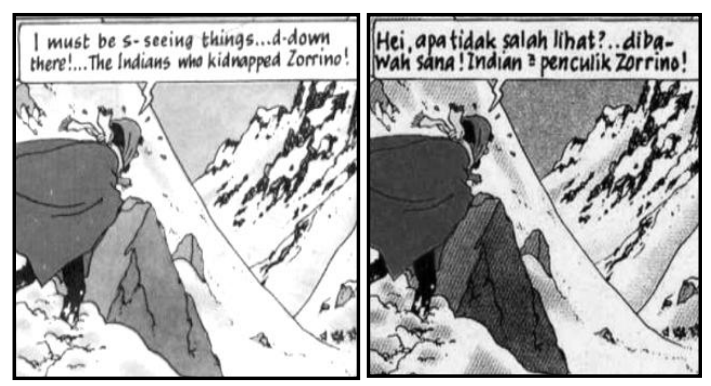

Figure 11: Unacceptable translation

In the example of Figure 10, it can be seen that there is a deletion of stuttering style in the TL. This decision result in the translation text being unacceptable. This is due to limitations of the dialog text column which requires the translator to do it.

\section{Readability}

Readability is the last aspect in the assessment of the translation quality. It also has 3 points of scales assessment, namely: high, medium, and low. Determining the value in this category does not always depend on the SL text. In other words, this assessment lies in the level of readability of the TL text whether it can easily be understood by the reader or not, as well as the existence of foreign terms that reduce the level of ease in reading the text. As shown in the Table 3, it can be seen that almost the entire data or about $96.30 \%$ shows that the translation quality has a high level of Readability. Only about $2.22 \%$ has a medium level and $1.48 \%$ shows a low level of 
readability. The distribution of the above points will be discussed further in the explanation below.

High readability

The translation results with a high level of readability if the translation is in match with the structure of the word, phrase, or pattern of sentence in TL and the dialogue is easy to be read. Although the meaning of SL is not the same as in TL, it will not give any significant effect on this assessment. The example can be seen in Figure 11.

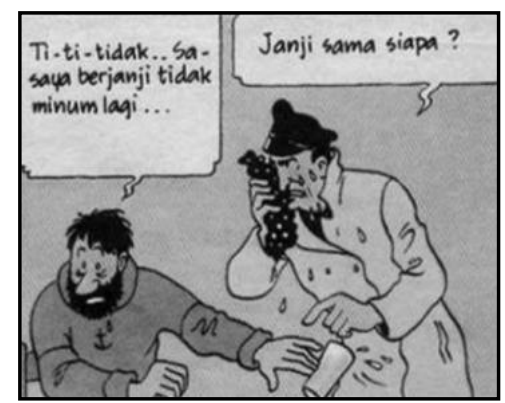

Figure 11: High readability

The text in Figure 11, all of the linguistic elements: stuttering in the form of repetitions or sentence structure in those spoken dialogue can be easily understood and read.

\section{Medium readability}

The assessment for this aspect, that the incorrect grammar and structure or emergence of foreign utterance borrowing results the TL text to be dificult to understand and read. This can be seen in Figure 12 .
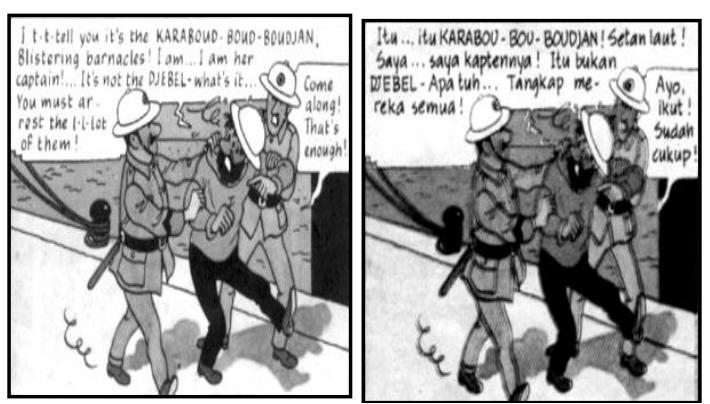

Figure 12: Medium readability

In he foreign utterance in Figure 12 occurs the stuttering stylecategorized as repetition. The word 'KARABOUDJIAN' is the name of the ship belonging to Captain Haddock. The foreign utterance is directly borrowed to the TL text. When observed from the lexical structure, this foreign utterance is remaining unknown andit has a diffcult spelling concept, seen from the grammatical arrangement of Indonesian language.

Low readability

The last category is a low level of readability. It has several meanings, such as: (1) the TL text does not have correct sentence structure; (2) almost the entire sentence-forming elements containing foreign utterance; (3) the deletion of linguistic elements in part or even the entire sentence in the TL text. The example can be seen in Figure 13.



Figure 13: Low readability

The example of Figure 13, where the sentence '... I'll sh-show him, the scarecrow.' from SL is not translated on TL. When observed on the TL text balloon, the number of words served almost filled the space out. Thus, the translator's decision to eliminate thissentenceare based on the limitation of word count in each text column.

\section{ANALYSIS}

This section disscuses the stuttering shifts spoken by Captain Haddock in The Adventure of Tintin comic series and its impact on translation quality. From the findings of this study, the relationship between domains with stuttering shift style that affects the translation quality value can be seen in the presentation of Table 4.

For the first stuttering initiator, the shift category shows that there are 72 out of 127 stuttering findings is Altered, which means more than half data findings can be categorized as a 
standart translation, considering that some of altered findings might have increased the quality of TL text and vice versa. While the stuttering which does not experienced a shift and of course categorized as a good translation are about 31 findings. Last, 22 data of total data findings are omitted. Thus, the assessments from overall findings on the Drunks are: 2.61 of accuracy (A); 2.63 of acceptability (Ac); and 2.92 of readability $(\mathrm{R})$. In other words, the value of the quality of accuracy and acceptability in this category is quite high, and even the readability aspect gets almost perfect value.

Table 4: Stuttering Shift Mapping toward Translation Quality

\begin{tabular}{llcccc}
\hline Domain & \multicolumn{2}{c}{$\begin{array}{c}\text { Shift } \\
\text { Mapping }\end{array}$} & \multicolumn{4}{c}{ Translation } \\
Quality \\
& \multicolumn{1}{c}{ Shift } & $\mathbf{\Sigma}$ & A & Ac & R \\
\hline Drunks & Altered & 72 & 2,40 & 2,4 & 2,82 \\
& Preserved & 31 & & & \\
& Omitted & 22 & & & \\
Situations & Altered & 24 & 2.75 & 2.84 & 2.9 \\
& Preserved & 1 & & & \\
Emotions & Omitted & 5 & & & \\
& Altered & 7 & 2.50 & 2.6 & 2.8 \\
& Preserved & 1 & & & \\
& Omitted & 2 & & & \\
\hline
\end{tabular}

Then in Situations, Stuttering which experienced a shift was 24 of the total 30 findings. As previously explained, the mapping of stuttering that undergoes a shift affects the quality of the translation in TL text. Then the second is stuttering which does not experience a shift is only one finding. And the last category is the omitted;thereare 5 findings. This omission however gives negative assesment or in other word decreases the translation quality value.

The last domain is Emotions, where the stuttering that experiences a shift is arround 7 of 10 total findings or about $70 \%$. Then stuttering which does not experience a shift is 1 or about $10 \%$. The last for omitted stuttering is found arround 2 pieces or about 20\%. Based on the data statistics in table 4 , it can be concluded that the stuttering utterance spoken by Captain Haddock in The Adventure of Tintin comic series has an ideal quality, both in terms of meaning and message accuracy; understandable grammatical complexity arrangement; and so for the natural use of language that can be easily read by readers.

In relation with previous researches, it is true that the phenomenon of stuttering or speech disfluency has been described as a form of identity of speakers in communication. Stuttering found in this study also shows that it is a representation of character identity with all the humour that is visualized through the creation of stuttering utterances spoken by Captain Haddock in this comic series. The stuttering shifts found mostly in the repetition category, this is in line with Tissi's (2000) study which states that the repetitions form is easy to divert into the TL text, because the form of repetitions is located at the beginning of sound on its lexical structure (letters, syllables, and words). For the shift that occurs, most of the shift findings are located at the lexical level, either in the form of shifting positions in the sentence structure (e.g the subject to object) or the word cl.ass (e.g. content tofunction words). However, this shift does not significantlyeffectthequality of the translation (aspects of acceptability and readability). This is because the shift is a creative form or stylistic effect of the translator in finding the equivalent message in the TL text. Then the element that reduces the translation quality is omission due to the non-transfered of the stuttering into the TL text; or it can also be a deletion of most or eventhe whole sentence in the dialogue spoken. 


\section{REFERENCES}

Alm, Per A. 2014. "Disorders Review: Stuttering in Relation to Anxiety, Temperament, and Personality: Review and Analysis with Focus on Causality." Journal of Fluency Disoreder: 2

Bakti, M. 2008. "Speech Disfluencies in Simultaneous Interpretation." Selected Papers of hte CETRA Research Seminar in Translation Studies Retrieved from $<$ https://www.arts.kuleuven.be/cetra/pa pers/papers.html>

Belyk, Michel, Shelly Jo Kraft, \& Brown, Steven. 2014. "Stuttering as a Trait or State: An ALE Meta-analysis of Neuroimaging Studies". European Journal of Neuroscience: 1

Catford, JC. 1965. A Linguistic Theory of Translation. Oxford: Oxford University Press

Gruyter, De. 2018. Translation Zone(s): A Stuttering: An Experiential Approach to Linguistic Hospitality, 1 (2): 164

Guitar, B. 2014. An Integrated Approach to Its Nature and Treatment (4th Edition). Philadelphia: Lippincott Williams \& Wilkins

Hollien, HG, C. Dejong, R. Martin, K. Schwartz \& Liljegren. 2001. "Effects of Ethanol Intoxication on Speech Suprasegmentals". Acoustical Society of American Journal, 110 (6): 3198-3206

Hough, J, Y. Tian, L. Ruiter, S. Betz, S.Kousidis, D. Schlangen \& J. Ginzburg. 2016. DUEL: A Multilingual Multimodal Dialogue Corpus for Disfluency, Exclamations, and Laughter. Retrieved from $<$ https://pub.unibielefeld.de/download/2 903080/2903084>

Johnson, W. 1959. The Onset of Stuttering. Minneapolis: University of Minnesota Press

McCloud, S. 1993. Understanding Comics: The Invisible Arts. New York: Kitchen Press
Miller, JF, S. Long, N. McKinley, S. Thormann, MA. Jones \& A. Nockerts. 2005 Language Sample Analysis II: The Wisconsin Guide. Madison: Wisconsin Department of Public Instruction: 1-8

Nababan, MR. 2008. Teori Menerjemah Bahasa Inggris. Yogyakarta: Pustaka Pelajar

Nababan, MR, A. Nuraeni, Sumardiono. 2012. "Pengembangan Model Penilaian Kualitas Terjemahan". Kajian Linguistik dan Sastra, 24 (1): 39-57

Neubert, A. 2004. "Case Studies in Translation: The Study of Translation." Across Language and Culture, 5 (1): 521

Nida, EA \& CR. Taber. 1974. The Theory and Practice of Translation. Leiden: The United Bible Societies

Plevoets, K \& B. Defrancq, 2016. "The Effect of Informational Load on Disfluencies in Interpreting." Translation and Interpreting Studies, 11 (2): 202-224

Remi, GP. The Adventure of Tintin: The Series. Retrieved from <http://www. tintinebook.com>

Remi, GP. Kisah-Kisah PetualanganTintin. Serial Komik. Retrieved from $<$ https://koleksi-tintin.blogspot.com>

Santosa, Riyadi. 2014. Metode Penelitian Kualitatif Kebahasaan. Surakarta:UNS Press

Spradley, James P. 1980. MetodeEtnografi. Yogyakarta: Tiara Wacana.

Sutopo, HB. 2006. Metodologi Penelitian Kualitatif. Surakarta: UNS Press

Tissi, B. 2000. "Silent Pauses and Disfluencies in Simultaneous Interpretation: A Descriptive Analysis.” The Interpreters' Newsletter, 10 Edizioni.Trieste: Università di Trieste

\section{ACKNOWLEDGEMENT}

This article is a part of author's S-2 thesis under the supervision of Prof. Drs. MR Nababan, M.Ed, MA, Ph.D and Prof. Dr. Djatmika, MA from Universitas Sebelas Maret Surakarta. 